\title{
KULEUVEN
}

\section{The IA|BE 2015 mortality projection for the Belgian population}

Katrien Antonio, Lize Devolder and Sander Devriendt 


\title{
The IA $\mid$ BE 2015 mortality projection for the Belgian population
}

\author{
Katrien Antonio ${ }^{* 1,2}$, Lize Devolder ${ }^{\dagger}$, and Sander Devriendt ${ }^{\ddagger}$ \\ ${ }^{1}$ Faculty of Economics and Business, KU Leuven, Belgium. \\ ${ }^{2}$ Faculty of Economics and Business, University of Amsterdam, The Netherlands.
}

February 25, 2015

\section{Introduction}

Life insurers, pension funds, health care providers and social security institutions face increasing expenses due to continuing improvements of mortality rates. The quantification of longevity risk in a systematic way requires stochastic mortality projection models, as Barrieu et al. (2012) indicate. The development of such models receives wide coverage in the actuarial, demographic and statistical literature, starting from the seminal work by Lee \& Carter ([LC]) (Lee and Carter (1992)). As a follow-up to the 2002 mortality study of the former KVBA-ARAB (now: Institute of Actuaries in Belgium [IA|BE]) (see Lambrechts (2001), Brouhns et al. (2002a), Delfosse and Boelen (2002)), this report presents the set-up and technical specifications of the 2015 mortality projection model of the Institute of Actuaries in Belgium. The report also documents the resulting projections and the use of this model in actuarial applications. The IA|BE 2015 mortality projection model is a fully stochastic projection model of Li \& Lee ([LL]) type, see $\mathrm{Li}$ and Lee (2005). The model is an application of the AG 2014 projection methodology of the Dutch Koninklijk Actuarieel Genootschap (see Koninklijk Actuarieel Genootschap (2014) ${ }^{1}$ ) to the Belgian setting. This multi-population model projects Belgian mortality rates using Belgian mortality data together with observed mortality statistics for a collection of European countries with similar socioeconomic characteristics. A Lee \& Carter model is imposed for the European mortality trend as well as for the Belgian deviation from this common trend. $\mathrm{We}^{2}$ have chosen this model as the preferred model from a comparative analysis of stochastic projection models proposed in recent scientific literature. As part of this comparative exercise we studied the single population mortality models from the papers by Cairns et al. (2009), Haberman and Renshaw (2011), Börger et al. (2014), Van Berkum et al. (2014), and the multi-population mortality models of Li and Lee (2005) and Koninklijk Actuarieel Genootschap (2014). The

\footnotetext{
${ }^{*}$ Corresponding author. E-mail address: katrien.antonio@kuleuven.be

${ }^{\dagger}$ Research assistant on the mortality project at KU Leuven, AFI Department, Insurance Research Group

${ }^{\ddagger}$ Research assistant on the mortality project at KU Leuven, AFI Department, Insurance Research Group

${ }^{1}$ The first author is a member of the Werkgroep Prognosetafel which developed the AG2014 projection model, together with the Commissie Sterfteonderzoek of the Dutch Koninklijk Actuarieel Genootschap. For the names of all members of the Werkgroep and Commissie, see 'Acknowledgements'.

${ }^{2}$ The authors, together with the members of the IA|BE working group on mortality (chair: Audrey Meganck).
} 
Cairns et al. (2006) stochastic two factor mortality model, and its generalizations proposed in Cairns et al. (2009), have not been investigated, since these Cairns, Blake \& Dowd ([CBD]) mortality models are designed for pensioner age mortality only, whereas the IA|BE study puts focus on a projection model for the full age range (starting from age 0). For each model in the study we carefully examined its calibration, the modelling and projection of time dependent parameters and the produced forecasts. We evaluated the collection of mortality models using the criteria proposed in Cairns et al. (2009), supplemented with our own points of attention regarding biological reasonableness, statistical performance and transparency. The preferred model, and its calibration methodology, follows the AG2014 projection methodology as described in Koninklijk Actuarieel Genootschap (2014). Readers may want to consult the Koninklijk Actuarieel Genootschap (2014) documentation as additional reading material.

This report is organized as follows. A technical description of the model is in Section 2. The subsections 2.1, 2.3 and 2.4 follow to a large extent Appendix A in Koninklijk Actuarieel Genootschap (2014). The use of the model and its applications are documented in Section 3. Section 4 concludes. Parameter estimates and the resulting IA|BE 2015 mortality projection for Belgium are available in an online appendix.

\section{Technical description of the model}

\section{$2.1 \quad$ Notation}

Let $\mathcal{X}$ denote a collection of ages and $\mathcal{T}$ a collection of years. We denote with $q_{x, t}$, for $x \in \mathcal{X}$ and $t \in \mathcal{T}$, the probability that a person who is alive at 1 January of year $t$, and who was born on 1 January of year $t-x$, will be death on 1 January of year $t+1$. We call $q_{x, t}$ the mortality rate at exact age $x$ in year $t$. The stochastic mortality models discussed in the papers mentioned in Section 1 directly model (a transformation of) $q_{x, t}$ or they model the force of mortality, $\mu_{x, t}$. Under the assumption of piecewise constant force of mortality, i.e. $\mu_{x+s, t+s}=\mu_{x, t}$ for $0 \leqslant s<1$, the following relation holds between $q_{x, t}$ and $\mu_{x, t}$ :

$$
q_{x, t}=1-\exp \left(-\mu_{x, t}\right) .
$$

Expression (1) enables switching from the force of mortality to the mortality rate, and vice versa, in a straightforward way. For more details about these concepts and additional reading material we refer to the monograph of Pitacco et al. (2009).

\subsection{Data}

We use data on the observed number of deaths, $d_{x, t}$, and the corresponding exposures to risk, $E_{x, t}$, as available in the Human Mortality Database ${ }^{3}$ ([HMD]). From this database we use the tables 'Deaths' and 'Exposure to risk' in $1 \times 1$ format. In our study we calibrate mortality models on age range $\mathcal{X}=\{0, \ldots, 90\}$ and calibration period $\mathcal{T}=\{1970, \ldots, 2013\}$. We use data from a collection of 14 European countries, namely Belgium, The Netherlands, Luxemburg, Norway, Switzerland, Austria, Ireland, Sweden, Denmark, West-Germany, Finland, Iceland, England \& Wales and France. All these countries have a Gross Domestic Product ([GDP]) per capita above

\footnotetext{
${ }^{3}$ This database is available at www.mortality.org.
} 
European average ${ }^{4}$. For Belgium we obtain data from 1970 until (and including) 2012 from HMD. For the other countries in the study we download data from 1970 until (and including) 2009 from HMD. We supplement the Belgian data with 2013 observations on deaths $\left(d_{x, 2013}^{\mathrm{BE}}\right)$ and exposures to risk $\left(E_{x, 2013}^{\mathrm{BE}}\right)$, as defined according to the protocol of $\mathrm{HMD}^{5}$. Adjusting to the HMD protocol requires the following transformations, where $d_{x, t}^{\mathrm{ADSEI}}$ refers to deaths obtained from $\mathrm{AD} \mathrm{SEI}^{6}$ and $p_{x, t}^{\mathrm{ADSEI}}$ refers to population size ${ }^{7}$ from the same source:

$$
\begin{aligned}
d_{x, t}^{\mathrm{BE}} & =\frac{1}{2} d_{x-1, t}^{\mathrm{ADSEI}}+\frac{1}{2} d_{x, t}^{\mathrm{ADSEI}} \text { if } x>0 \\
d_{0, t}^{\mathrm{BE}} & =d_{\mathrm{birth}, t}^{\mathrm{ADSEI}}+\frac{1}{2} d_{0, t}^{\mathrm{ADSEI}},
\end{aligned}
$$

and $^{8}$

$$
\begin{aligned}
E_{x, t}^{\mathrm{BE}} & =\frac{1}{2}\left(p_{x, t}^{\mathrm{ADSEI}}+p_{x, t+1}^{\mathrm{ADSEI}}\right)+\frac{1}{6}\left(\frac{1}{2} d_{x-1, t}^{\mathrm{ADSEI}}-\frac{1}{2} d_{x, t}^{\mathrm{ADSEI}}\right) \text { if } x>0 \\
E_{0, t}^{\mathrm{BE}} & =\frac{1}{2}\left(p_{0, t}^{\mathrm{ADSEI}}+p_{0, t+1}^{\mathrm{ADSEI}}\right)+\frac{1}{6}\left(d_{\mathrm{birth}, t}^{\mathrm{ADSEI}}-\frac{1}{2} d_{0, t}^{\mathrm{ADSEI}}\right) .
\end{aligned}
$$

As such, we proceed with a data set of deaths and exposures, as defined according to the HMD protocol, for a collection of 13 European countries during 1970-2009 and for Belgium during 1970-2013. We extract this data source for males and females. The unisex data are created by aggregating the data for males and females.

\subsection{Model specification}

The mortality model specifies the logarithm of the force of mortality for Belgium, $\mu_{x, t}$, as follows

$$
\begin{aligned}
& \ln \mu_{x, t}=\ln \mu_{x, t}^{\mathrm{EU}}+\ln \mu_{x, t}^{\mathrm{BE}} \\
& \ln \mu_{x, t}^{\mathrm{EU}}=A_{x}+B_{x} K_{t} \\
& \ln \mu_{x, t}^{\mathrm{BE}}=\alpha_{x}+\beta_{x} \kappa_{t} .
\end{aligned}
$$

We recognize two times a Lee \& Carter specification; (3) is a LC model for the European evolution of mortality (driven by $\mu_{x, t}^{\mathrm{EU}}$ ) and (4) is a LC model for the Belgian deviation from this common trend (specified by $\mu_{x, t}^{\mathrm{BE}}$ ). We calibrate this model on data with ages ranging from 0 up to 90 , thus $\mathcal{X}=\{0, \ldots, 90\}$, and years from 1970 up to 2013 , thus $\mathcal{T}=\{1970, \ldots, 2013\}$.

\footnotetext{
${ }^{4}$ Source: World Bank Data for 2013 on GDP per capita in US dollar, http://data.worldbank.org/ indicator/NY.GDP.PCAP.CD. The GDP per capita for the Euro area is 39,360 USD in 2013 and the fourteen countries listed are the countries in Europe with a higher GDP per capita.

${ }^{5}$ This protocol is available from http://www.mortality.org/Public/Docs/MethodsProtocol.pdf.

${ }^{6} \mathrm{AD}$ SEI is Algemene Directie Statistiek en Economische Informatie. Number of deaths are available at http://statbel.fgov.be/nl/modules/publications/statistiques/bevolking/downloads/bevolking_ sterftetafels.jsp, where we used Sterftetafels jaarlijks in verstreken leeftijd (1997-2013) and the data $d_{x}$ from this table.

${ }^{7}$ We use the $p_{x}$ column in the http://statbel.fgov.be/nl/modules/publications/statistiques/ bevolking/downloads/bevolking_sterftetafels.jsp data source (up to 2013). For the 2014 data we use http://www .plan.be/databases/database_det.php?lang=fr\&ID=35.

${ }^{8}$ See formula (49) in the HMD protocol.
} 
The calibration methodology is described in Section 2.4. For the time dependent parameters, $K_{t}$ and $\kappa_{t}$, the following time series models are used

$$
\begin{aligned}
K_{t+1} & =K_{t}+\theta+\varepsilon_{t+1} \\
\kappa_{t+1} & =a \kappa_{t}+\delta_{t+1} .
\end{aligned}
$$

The dynamics of the common period effect (see (5)), $K_{t}$, are modelled with a Random Walk with Drift ([RWD]), where $\theta$ is the drift and $\varepsilon_{t+1}$ is white noise. The Belgian period effect (see (6)), $\kappa_{t}$, follows an AR(1) process without intercept. We calibrate the parameters in these time series specifications on the estimated $K_{t}$ and $\kappa_{t}$ parameters, for $t \in \mathcal{T}$, and use these dynamics to forecast $\mu_{x, t}$ for $t \in\{2014,2015, \ldots, 2060\}$. This projection strategy is documented in Section 2.5.

\subsection{Calibration}

We calibrate the parameters $\left(A_{x}, B_{x}, K_{t}, \alpha_{x}, \beta_{x}\right.$ and $\left.\kappa_{t}\right)$ in the LL specification using Maximum Likelihood Estimation ([MLE]). Following the seminal paper by Brouhns et al. (2002b) we assume a Poisson distribution for the number of deaths random variable $D_{x t}$, with mean $E_{x, t} \cdot \mu_{x, t}$ and $E_{x, t}$ the observed exposure to risk. To avoid identification problems in the LL model we use a conditional maximum likelihood approach as in Li (2013) and Koninklijk Actuarieel Genootschap (2014). We calibrate the common parameters (i.e. $A_{x}, B_{x}$ and $K_{t}$ ) in a first step, followed by the calibration of the Belgian parameters (i.e. $\alpha_{x}, \beta_{x}$ and $\kappa_{t}$ ) in a second step.

1. Observed deaths to calibrate the European trend, $d_{x, t}^{\mathrm{EU}}$, and corresponding exposures to risk, $E_{x, t}^{\mathrm{EU}}$, are obtained from the Human Mortality Database (see Section 2.2) by aggregating deaths and exposures over the 14 selected countries, using $\mathcal{X}=\{0, \ldots, 90\}$ and $\overline{\mathcal{T}}=\{1970, \ldots, 2009\}$. We maximize the following Poisson likelihood

$$
\max _{A_{x}, B_{x}, K_{t}} \prod_{x \in \mathcal{X}} \prod_{t \in \mathcal{\mathcal { T }}}\left(E_{x t}^{\mathrm{EU}} \mu_{x, t}^{\mathrm{EU}}\right)^{d_{x t}^{\mathrm{EU}}} \cdot \exp \left(-E_{x t}^{\mathrm{EU}} \mu_{x, t}^{\mathrm{EU}}\right) /\left(d_{x t}^{\mathrm{EU} !}\right),
$$

with $\mu_{x, t}^{\mathrm{EU}}=\exp \left(A_{x}+B_{x} K_{t}\right)$. We apply the usual Lee \& Carter parameter constraints to identify parameters in a unique way, namely $\sum_{t \in \overline{\mathcal{T}}} K_{t}=0$ and $\sum_{x \in \mathcal{X}} B_{x}=1$.

2. For Belgium, in contrast to the other European countries used in the model calibration, we have data up to 2013 and want to use the most recent set of data when calibrating the mortality model. Therefore, we extend the parameter estimates for $K_{t}$ to the years $\{2010,2011,2012,2013\}$ using linear extrapolation, namely

$$
K_{2009+s}=K_{2009}+s\left(K_{2009}-K_{1970}\right) /(2009-1970),
$$

with $s \in\{1,2,3,4\}$. The linear extrapolation is justified by the linear pattern of the estimated $K_{t}$ parameters, see for example Figure 1 (right plot).

3. We calibrate the parameters for Belgium (i.e. $\alpha_{x}, \beta_{x}$ and $\kappa_{t}$ ) by maximizing the following Poisson likelihood, conditional on the common parameters estimated in step 1 of this procedure. Thus,

$$
\max _{\alpha_{x}, \beta_{x}, \kappa_{t}} \prod_{x \in \mathcal{X}} \prod_{t \in \mathcal{T}}\left(E_{x t}^{\mathrm{BE}} \mu_{x, t}\right)^{d_{x t}^{\mathrm{BE}}} \cdot \exp \left(-E_{x t}^{\mathrm{BE}} \mu_{x, t}\right) /\left(d_{x t}^{\mathrm{BE} !)},\right.
$$


where $\mu_{x, t}=\mu_{x, t}^{\mathrm{EU}} \cdot \exp \left(\alpha_{x}+\beta_{x} \kappa_{t}\right)$. We calibrate the parameters for Belgium on ages $\mathcal{X}=\{0, \ldots, 90\}$ and years $t \in \mathcal{T}=\{1970, \ldots, 2013\}$. Once again we normalize the estimated parameters by imposing $\sum_{t \in \mathcal{T}} \kappa_{t}=0$ and $\sum_{x \in \mathcal{X}} \beta_{x}=1$.

We apply this calibration strategy ${ }^{9}$ separately for male, female and unisex data. We illustrate the resulting parameter estimates for female data in Figure 1 (common parameters) and Figure 2 (Belgian parameters). An online appendix to this report lists all parameter estimates.
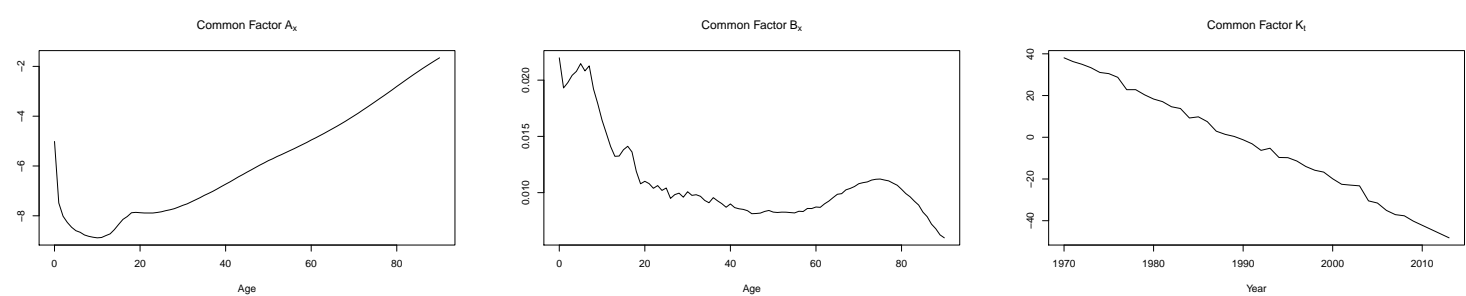

Figure 1: Estimated common parameters, female data, ages 0-90, years 1970-2009: $A_{x}, B_{x}$ and $K_{t}$.
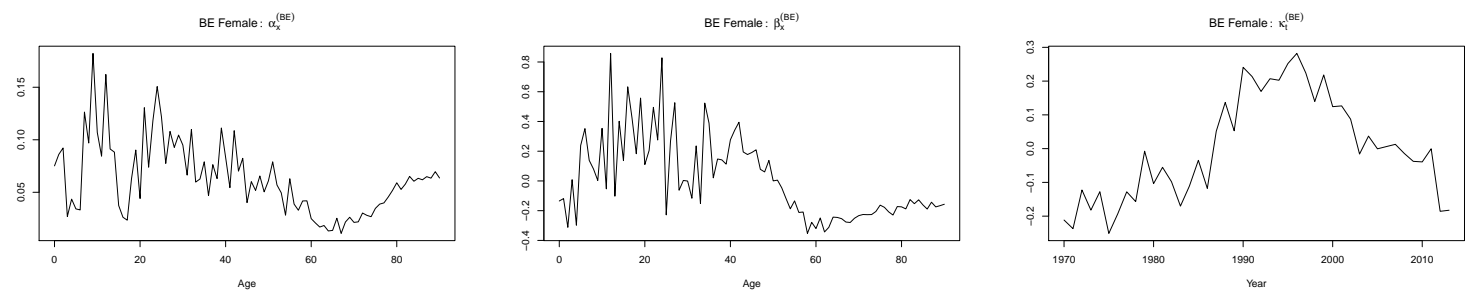

Figure 2: Estimated parameters for Belgium, female data, ages 0-90, years 1970-2013: $\alpha_{x}, \beta_{x}$ and $\kappa_{t}$.

\subsection{Projection}

Calibrating the time series models. The mortality model specified in Section 2.4 together with the time dynamics specified in (5) and (6) allows to generate future scenarios of mortality. First, we calibrate the time series models to the parameter estimates $\left\{\left(K_{t}, \kappa_{t}\right) \mid t \in \mathcal{T}\right\}$ with $\mathcal{T}=$ $\{1970, \ldots, 2013\}$. We hereby assume a bivariate normal distribution for the error terms $\left(\varepsilon_{t}, \delta_{t}\right)$ with mean $(0,0)$ and covariance matrix $C$. The error terms are independent and identically distributed for all $t$. The parameters $\theta, a$ and $C$, used in the time series specifications, are estimated using maximum likelihood ${ }^{10}$. The resulting parameter estimates (for males, females and unisex data) are listed in Table 1 (males and females) and 2 (unisex).

Generating future scenarios of mortality. Future mortality scenarios can be generated using the following step by step approach. For each future scenario $i=1, \ldots, N$, and with $t$ running from 2014 to some specific end year $T$, we use the following strategy.

\footnotetext{
${ }^{9}$ We use routines written in $\mathrm{R}$.

${ }^{10}$ In $R$ we use Seemingly Unrelated Regression through the package systemfit, we use the function systemfit with options method="SUR" and methodResidCov="noDfCor".
} 


\begin{tabular}{llllllll}
\hline \multicolumn{4}{c}{ males } & \multicolumn{3}{c}{ females } \\
\hline$\theta$ & $a$ & \multicolumn{2}{c}{$C$} & $\theta$ & $a$ & \multicolumn{2}{c}{$C$} \\
-2.0372 & 0.9996 & 1.7603 & -0.1584 & -2.0029 & 0.8645 & 2.4939 & -0.01895 \\
& & -0.1584 & 0.0649 & & & -0.01895 & 0.0057 \\
\hline
\end{tabular}

Table 1: Time series parameter estimates for data on males and females.

\begin{tabular}{llll}
\hline \multicolumn{4}{c}{ unisex } \\
\hline$\theta$ & $a$ & \multicolumn{2}{c}{$C$} \\
-2.0161 & 0.9229 & 2.0820 & -0.0298 \\
& & -0.0298 & 0.0184 \\
\hline
\end{tabular}

Table 2: Time series parameter estimates for unisex data.

1. We simulate future $\left(K_{t}^{i}, \kappa_{t}^{i}\right)$ using the time dynamics specified in (5) and (6), with parameter estimates as listed in Table 1 or 2 . We hereby start with $\left(K_{2013}, \kappa_{2013}\right)$ as obtained with the calibration strategy from Section 2.4. These values are listed in the online appendix. We generate $\left(\varepsilon_{t}, \delta_{t}\right)$ from a bivariate normal distribution with mean $(0,0)$ and covariance matrix $C$, as listed in Table 1 or 2 .

2. Using the simulated $\left(K_{t}^{i}, \kappa_{t}^{i}\right)$ for $t=2014, \ldots, T$, we obtain $\mu_{x, t}^{i}$ using (2), (3) and (4) and the age specific parameters $\left(A_{x}, \alpha_{x}, B_{x}, \beta_{x}\right)$.

Figure 3 illustrates the projection of the time dependent parameters $K_{t}$ (left) and $\kappa_{t}$ (right) for females. We generate 10,000 scenarios and show the corresponding fan charts (formed by the median, $0.5 \%$ and $99.5 \%$ quantiles).
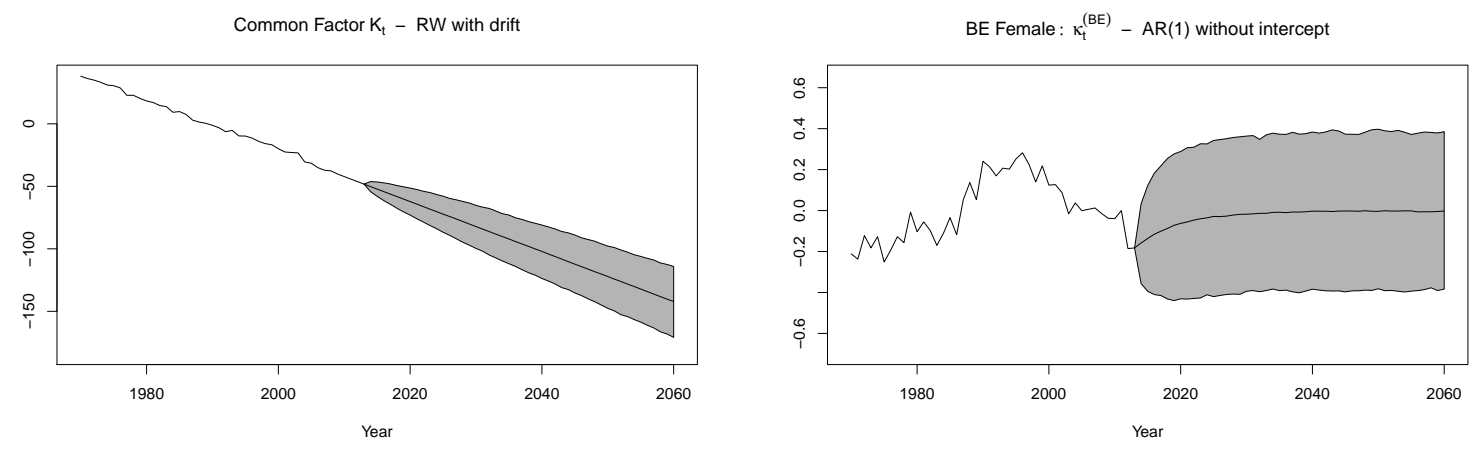

Figure 3: Projection of time dependent parameters: $K_{t}$ and $\kappa_{t}$, female data. We plot $0.5 \%$ quantile, median and $99.5 \%$ quantile obtained from 10,000 simulations.

Closing for old ages. We use Kannistö (1992) to close each mortality scenario for old ages, say $x \in\{91,92, \ldots, 120\}$. This mortality law is chosen from a comparative analysis of techniques 
to close mortality tables, documented in Antonio (2012). This parametric law specifies the force of mortality in each scenario $i$, for ages $x>90$ and a specific year $t$, as follows:

$$
\mu_{x, t}^{i}=\frac{\phi_{1}^{i, t} \exp \left(\phi_{2}^{i, t} x\right)}{1+\phi_{1}^{i, t} \exp \left(\phi_{2}^{i, t} x\right)} .
$$

We estimate $\left(\phi_{1}^{i}, \phi_{2}^{i}\right)$ for each scenario $i$ and year $t$ using the relation (see Doray (2008))

$$
\operatorname{logit} \mu_{x, t}^{i}=\log \left(\phi_{1}^{i, t}\right)+\phi_{2}^{i, t} x
$$

which we estimate with OLS on the ages $x \in\{80,81, \ldots, 90\}$. The estimates for $\left(\phi_{1}^{i, t}, \phi_{2}^{i, t}\right)$ are then used in (9) to close the generated mortality scenario for ages $x>90$.

Finally, we can switch to scenarios for future mortality rates using the transformation in (1), thus

$$
q_{x, t}^{i}=1-\exp \left(-\mu_{x, t}^{i}\right),
$$

for $t=2014,2015, \ldots, T$ and $x \in 0,1, \ldots, 120$.

\section{Results and applications}

\subsection{Fitted and simulated mortality rates}

Figure 4 shows the calibration of $q_{x, t}$ for Belgian females and a selection of ages $x \in\{25,45,65,85\}$. Figure 5 shows the corresponding results for males. We show the median and $99 \%$ pointwise confidence intervals based on 10,000 scenarios of projected mortality rates. The black dots in this Figure are the observed mortality rates $q_{x, t}$. The dotted line indicates the mortality rates fitted with the model specified in Section 2.3. As a reference line we add the projection 2013-2060 of Federaal Planbureau $(2014)^{11}$. The $q_{x, t}$ in the FPB table are defined at age in completed years and have been transformed to $q_{x, t}$ 's at exact age, using the transformation documented in Jaumain and Vandeschrick $(2012)^{12}$. The FPB model is a deterministic model, and its calibration does not follow the nowadays standard assumption of Poisson likelihood for the number of deaths. The projection of FPB is using calibration period 1991-2012 ${ }^{13}$, whereas earlier projections of the FPB were using a calibration period starting in $1970^{14}$.

\subsection{The IA|BE 2015 mortality projection for Belgium}

The projected mortality table obtained with the best estimates of future $\left(K_{t}, \kappa_{t}\right)$ (with $t=$ $2014,2015, \ldots, 2060)$ is the resulting 'IA|BE 2015 mortality projection for the Belgian population'. These best estimates for the period effects result from (5) and (6) with noise terms $\varepsilon_{t}=0=\delta_{t}$ for all future $t$. We close the mortality table obtained in this way with Kannistö (1992) such that $\mu_{x, t}$ for $x \in\{0,1, \ldots, 120\}$ and $t \in\{2014, \ldots, 2060\}$ result. The corresponding mortality rates $q_{x, t}$ follow from (1). This table is online available for males, females and as a unisex table.

\footnotetext{
${ }^{11} \mathrm{We}$ use the Prospectieve sterftequotiënten 2013-2060 available on http://www.plan.be/databases/ database_det.php?lang=nl\&ID=50 (tab QxCalc-F and QxCalc-M). The methodology is described in Federaal Planbureau (2009).

${ }^{12}$ We use formula (6) and (9) on page 11 of Jaumain and Vandeschrick (2012).

${ }^{13}$ See Federaal Planbureau (2014) on page 14.

${ }^{14}$ See Federaal Planbureau (2009) on page 2.
} 

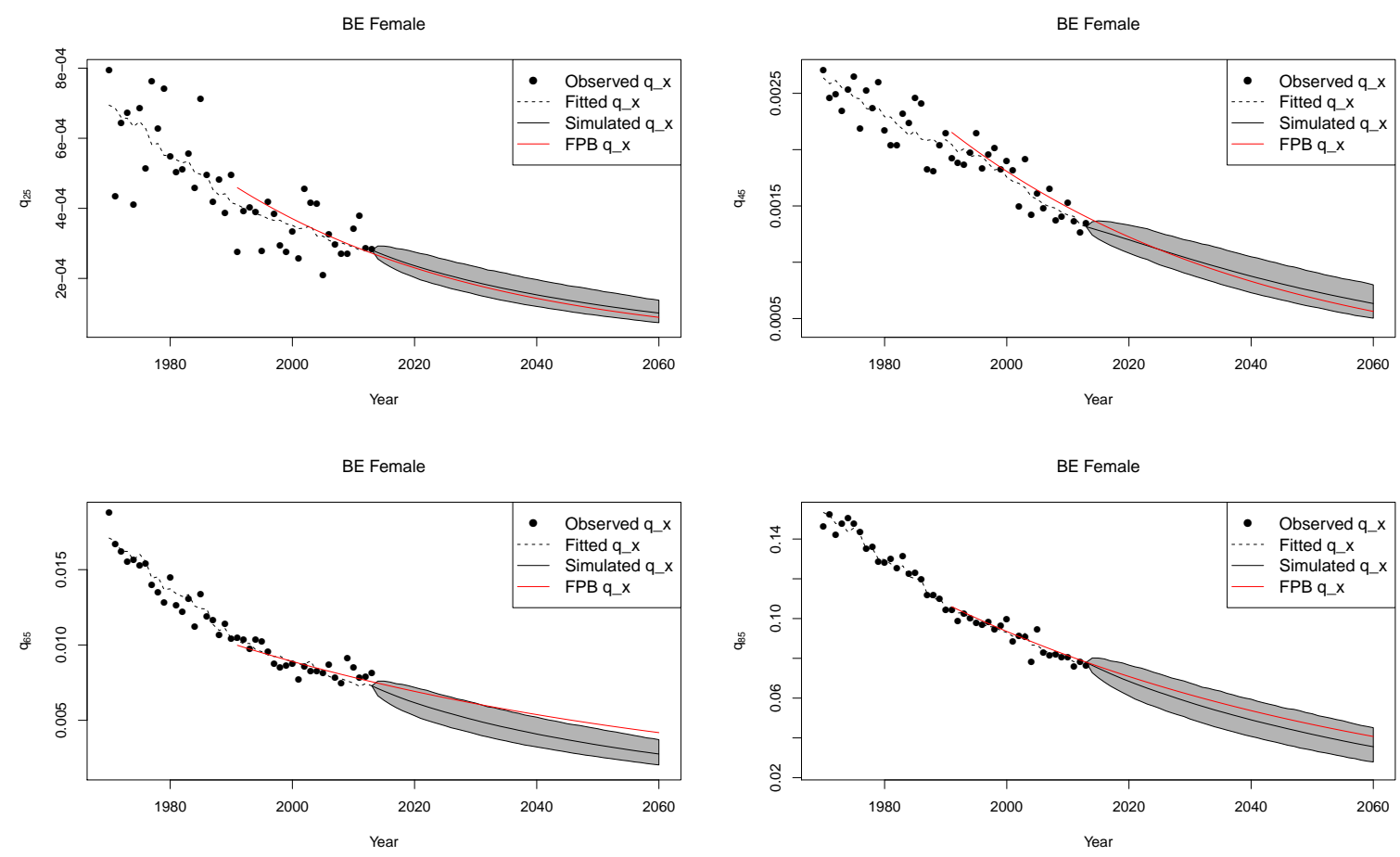

Figure 4: Estimated and projected mortality rates, $q_{x, t}$, for Belgium, female data, ages 25, 45 (top row) and 65,85 (bottom row). We plot $0.5 \%$ quantile, median and $99.5 \%$ quantile obtained from 10,000 simulations.

\subsection{Life expectancy: period and cohort}

From the simulated scenarios for future mortality rates we obtain simulations for the period as well as cohort life expectancy. Using the assumption of piecewise constant force of mortality, the period life expectancy for an $x$ year old in year $t$ is

$$
e_{x}^{\mathrm{per}}(t)=\frac{1-\exp \left(-\mu_{x, t}\right)}{\mu_{x, t}}+\sum_{k \geqslant 1}\left(\prod_{j=0}^{k-1} \exp \left(-\mu_{x+j, t}\right)\right) \frac{1-\exp \left(-\mu_{x+k, t}\right)}{\mu_{x+k, t}},
$$

and the cohort life expectancy for an $x$ year old in year $t$ is

$$
e_{x}^{\operatorname{coh}}(t)=\frac{1-\exp \left(-\mu_{x, t}\right)}{\mu_{x, t}}+\sum_{k \geqslant 1}\left(\prod_{j=0}^{k-1} \exp \left(-\mu_{x+j, t+j}\right)\right) \frac{1-\exp \left(-\mu_{x+k, t+k}\right)}{\mu_{x+k, t+k}},
$$

see, for example, Pitacco et al. (2009). Using the mortality scenarios generated as described in Section 2.5 we obtain simulations of the period and cohort expectancy, say $e_{x}^{\text {per, } i}(t)$ and $e_{x}^{\text {coh, } i}(t)$. Scenarios can be generated beyond 2060. Thus, when calculating life expectancies in, for example, 2060, we will project the mortality rates for 120 years after 2060. Of course, the best estimate table introduced in Section 3.2 can also be used to calculate a point estimate of the period and cohort life expectancy.

Figure 6 shows the observed period life expectancy (black dots) for a 0 (left) and 65 (right) year old female, the calibrated period life expectancy (red line) and the simulations of $e_{0}(t)$ and 

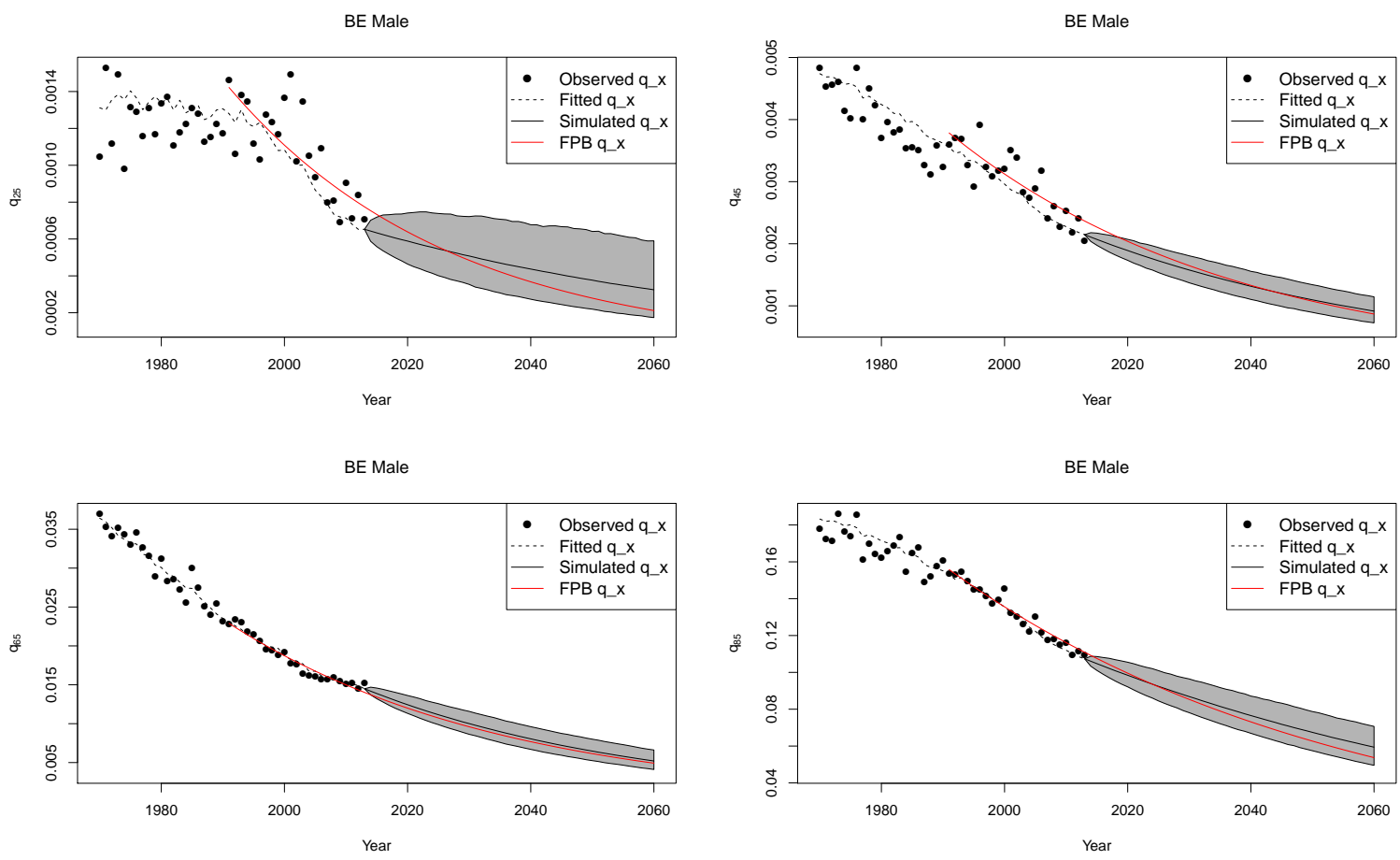

Figure 5: Estimated and projected mortality rates, $q_{x, t}$, for Belgium, male data, ages 25, 45 (top row) and 65,85 (bottom row). We plot $0.5 \%$ quantile, median and $99.5 \%$ quantile obtained from 10,000 simulations.

$e_{65}(t)$ for $t=2014, \ldots, 2024$. The blue fan chart shows the cohort life expectancy, $e_{0}^{\text {coh }}(t)$ and $e_{65}^{\text {coh }}(t)$. The corresponding results for males are in Figure 7.
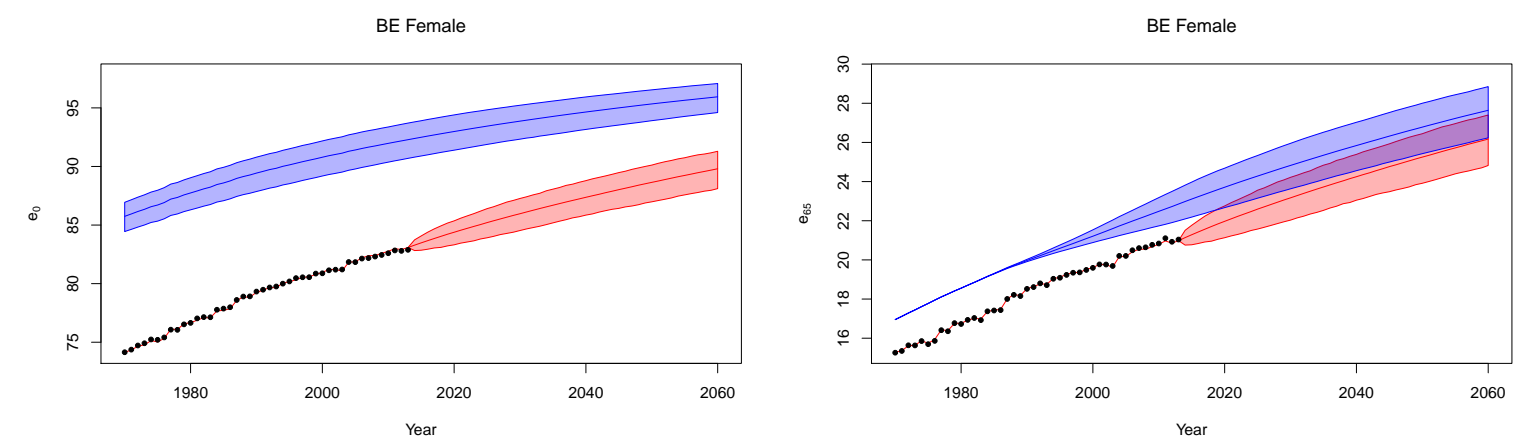

Figure 6: Period (black dots and red lines) and cohort (blue) life expectancy for a 0 year old (left) and 65 year old (right), female data. We plot $0.5 \%$ quantile, median and $99.5 \%$ quantile obtained from 10,000 simulations.

Tables 3 and 4 list the median and $99 \%$ confidence intervals of $e_{x}^{\text {coh }}(t)$ for some specific choice of $x$ and $t$. As a benchmark the table also shows the cohort life expectancies published by Federaal Planbureau $(2014)^{15}$. We transform these from ages in completed years to exact ages, using

\footnotetext{
${ }^{15}$ These are the EGxCalc in http://www.plan.be/databases/database_det.php?lang=nl\&ID=50.
} 

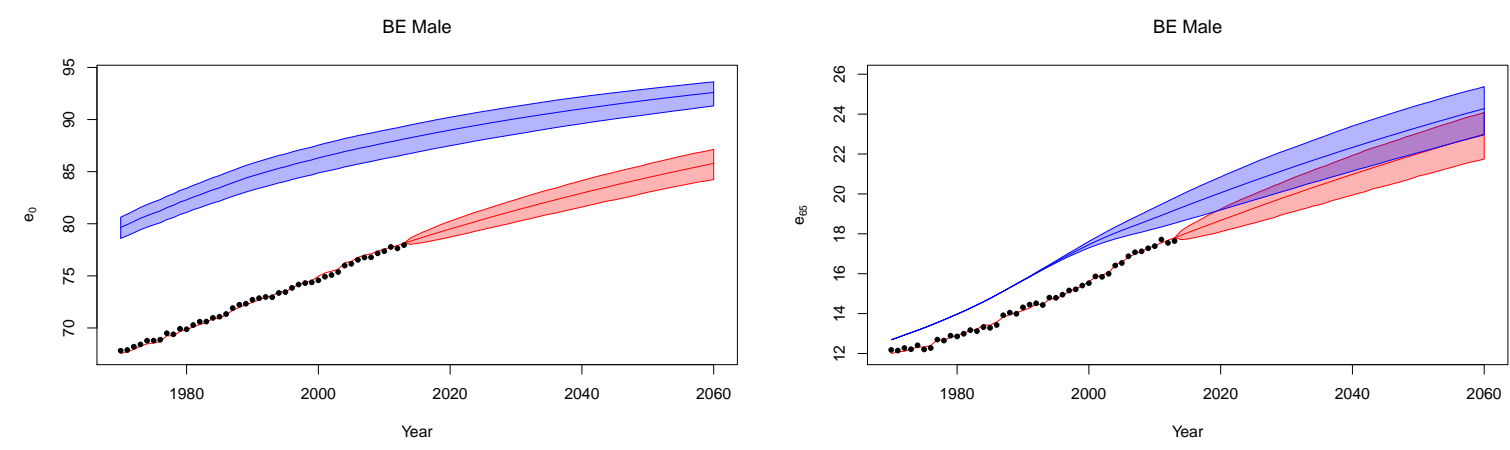

Figure 7: Period (black dots and red lines) and cohort (blue) life expectancy for a 0 year old (left) and 65 year old (right), male data. We plot $0.5 \%$ quantile, median and $99.5 \%$ quantile obtained from 10,000 simulations.

Jaumain and Vandeschrick $(2012)^{16}$.

\begin{tabular}{llcccc}
\hline year & \multicolumn{2}{c}{ males } & \multicolumn{2}{c}{ females } \\
& & & 65 & 0 & 65 \\
\hline 2014 & Best Est. & 88.26 & 19.3 & 92.4 & 22.98 \\
& {$\left[q_{0.5} ; q_{50} ; q_{99.5}\right]$} & {$[86.74 ; 88.26 ; 89.48]$} & {$[18.63 ; 19.31 ; 19.96]$} & {$[90.8 ; 92.39 ; 93.81]$} & {$[22.09 ; 22.98 ; 23.82]$} \\
& FPB & $(88.38 ; 88.58)$ & $(19.13 ; 19.57)$ & $(90.42 ; 90.67)$ & $(22.23 ; 22.7)$ \\
\hline 2040 & Best Est. & 91.04 & 22.33 & 94.66 & 25.85 \\
& {$\left[q_{0.5} ; q_{50} ; q_{99.5}\right]$} & {$[89.61 ; 91.03 ; 92.19]$} & {$[21.15 ; 22.33 ; 23.4]$} & {$[93.16 ; 94.65 ; 95.93]$} & {$[24.56 ; 25.84 ; 27.03]$} \\
& FPB & $(90.94 ; 91.31)$ & $(22.56 ; 23.03)$ & $(92.41 ; 92.81)$ & $(24.72 ; 25.2)$ \\
\hline 2060 & Best Est. & 92.61 & 24.28 & 95.95 & 27.64 \\
& {$\left[q_{0.5} ; q_{50} ; q_{99.5}\right]$} & {$[91.31 ; 92.6 ; 93.62]$} & {$[22.96 ; 24.28 ; 25.38]$} & {$[94.6 ; 95.95 ; 97.08]$} & {$[26.23 ; 27.64 ; 28.85]$} \\
& FPB & $(92.36 ; 92.78)$ & $(24.59 ; 25.07)$ & $(93.66 ; 94.1)$ & $(26.33 ; 26.81)$ \\
\hline
\end{tabular}

Table 3: Cohort life expectancy for a 0 and 65 year old, Best Estimate and $0.5 \%$ quantile, median and $99.5 \%$ quantile obtained from 10,000 simulations, males and females. 'FPB' refers to Federaal Planbureau (2014); the first number is the cohort life expectancy as published in Federaal Planbureau (2014) (i.e. using ages in completed years) and the second number is the cohort life expectancy at exact ages, using Jaumain and Vandeschrick (2012).

\subsection{Back testing the mortality model}

One of the evaluation criteria used in the process of selecting a mortality model, is the performance of a model in back tests (see Cairns et al. (2009)). We illustrate the performance of the IA|BE 2015 mortality projection model in two types of back tests. In Figure 8 (females) and 9 (males) we calibrate the model on data from 1970 to 2000 and use it to project mortality rates $q_{x, t}$ with $t \in\{2001, \ldots, 2013\}$.

Figure 10 and 11 visualize the results of three back tests, on calibration periods $\{1970, \ldots, 1980\}$, $\{1970, \ldots, 1990\}$ and $\{1970, \ldots, 2000\}$, expressed as fitted values and projections of the period life expectancy for a 0 year old and a 65 year old.

\footnotetext{
${ }^{16}$ We use formulas (11) and (14).
} 


\begin{tabular}{llcc}
\hline year & & \multicolumn{2}{c}{ unisex } \\
& & \multicolumn{1}{c}{0} & 65 \\
\hline 2014 & Best Est. & 90.78 & 21.26 \\
& {$\left[q_{0.5} ; q_{50} ; q_{99.5}\right]$} & {$[89.08 ; 90.78 ; 92.25]$} & {$[20.51 ; 21.26 ; 22.03]$} \\
& FPB 2014 & $(89.39 ; 89.62)$ & $(20.74 ; 21.19)$ \\
\hline 2040 & Best Est. & 93.46 & 24.37 \\
& {$\left[q_{0.5} ; q_{50} ; q_{99.5}\right]$} & {$[91.9 ; 93.47 ; 94.85]$} & {$[23.07 ; 24.36 ; 25.56]$} \\
& FPB 2014 & $(91.68 ; 92.06)$ & $(23.66 ; 24.13)$ \\
\hline 2060 & Best Est. & 95.02 & 26.35 \\
& {$\left[q_{0.5} ; q_{50} ; q_{99.5}\right]$} & {$[93.6 ; 95.02 ; 96.25]$} & {$[24.92 ; 26.35 ; 27.6]$} \\
& FPB 2014 & $(93.02 ; 93.45)$ & $(25.47 ; 25.95)$ \\
\hline
\end{tabular}

Table 4: Cohort life expectancy for a 0 and 65 year old, Best Estimate and $0.5 \%$ quantile, median and $99.5 \%$ quantile obtained from 10,000 simulations, unisex data. 'FPB' refers to Federaal Planbureau (2014); the first number is the cohort life expectancy as published in Federaal Planbureau (2014) (i.e. using ages in completed years) and the second number is the cohort life expectancy at exact ages, using Jaumain and Vandeschrick (2012).
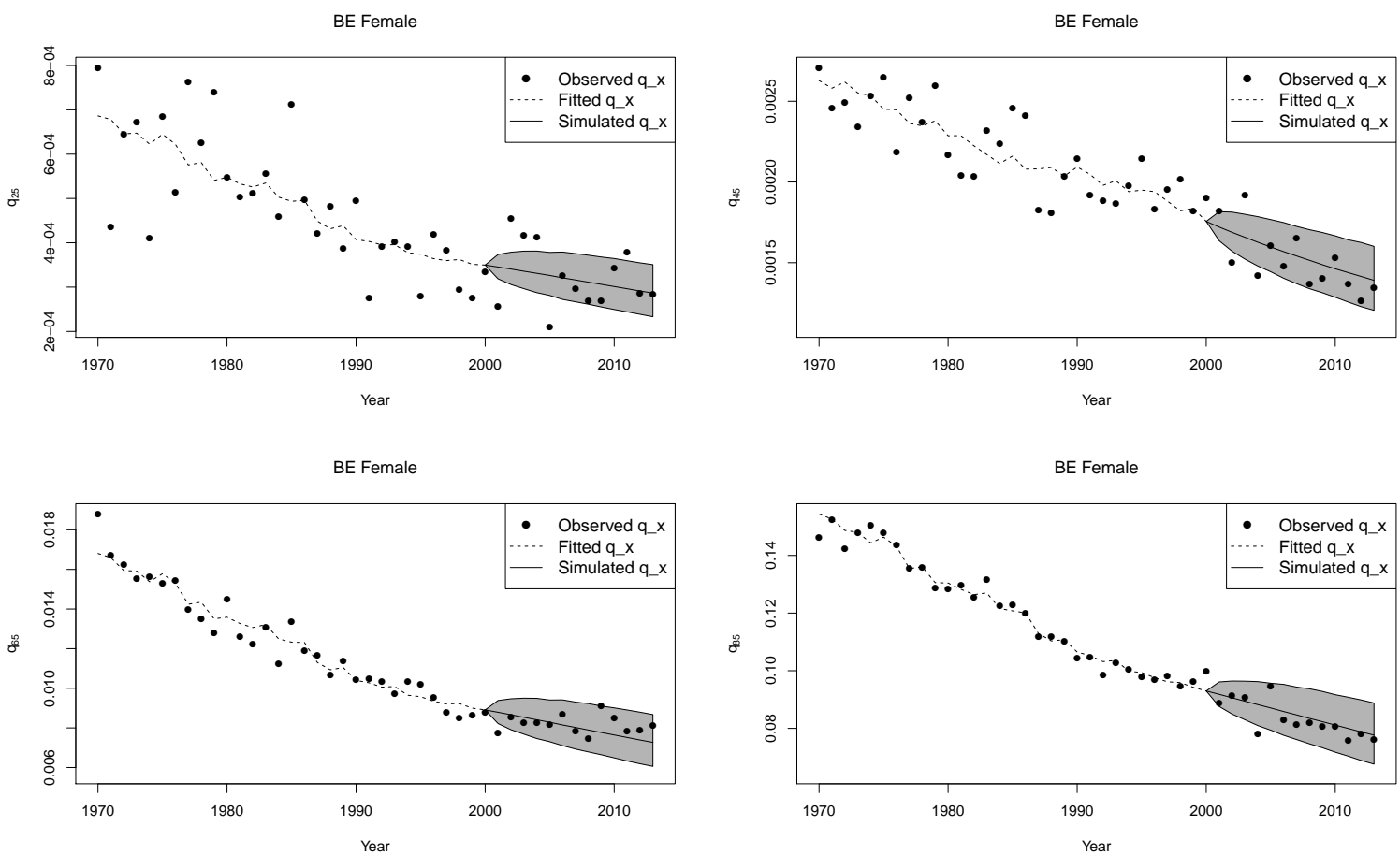

Figure 8: Back test on $q_{x, t}$, for Belgium, female data, ages 25, 45 (top row) and 65, 85 (bottom row). We calibrate on 1970-2000 data and then project 2001-2013. We plot $0.5 \%$, median and $99.5 \%$ quantile obtained from 10,000 simulations.

\section{Conclusion}

This report describes the methodology used to produce the IA|BE 2015 mortality projection as well as the stochastic model underneath this best estimate table. The mortality model is of 

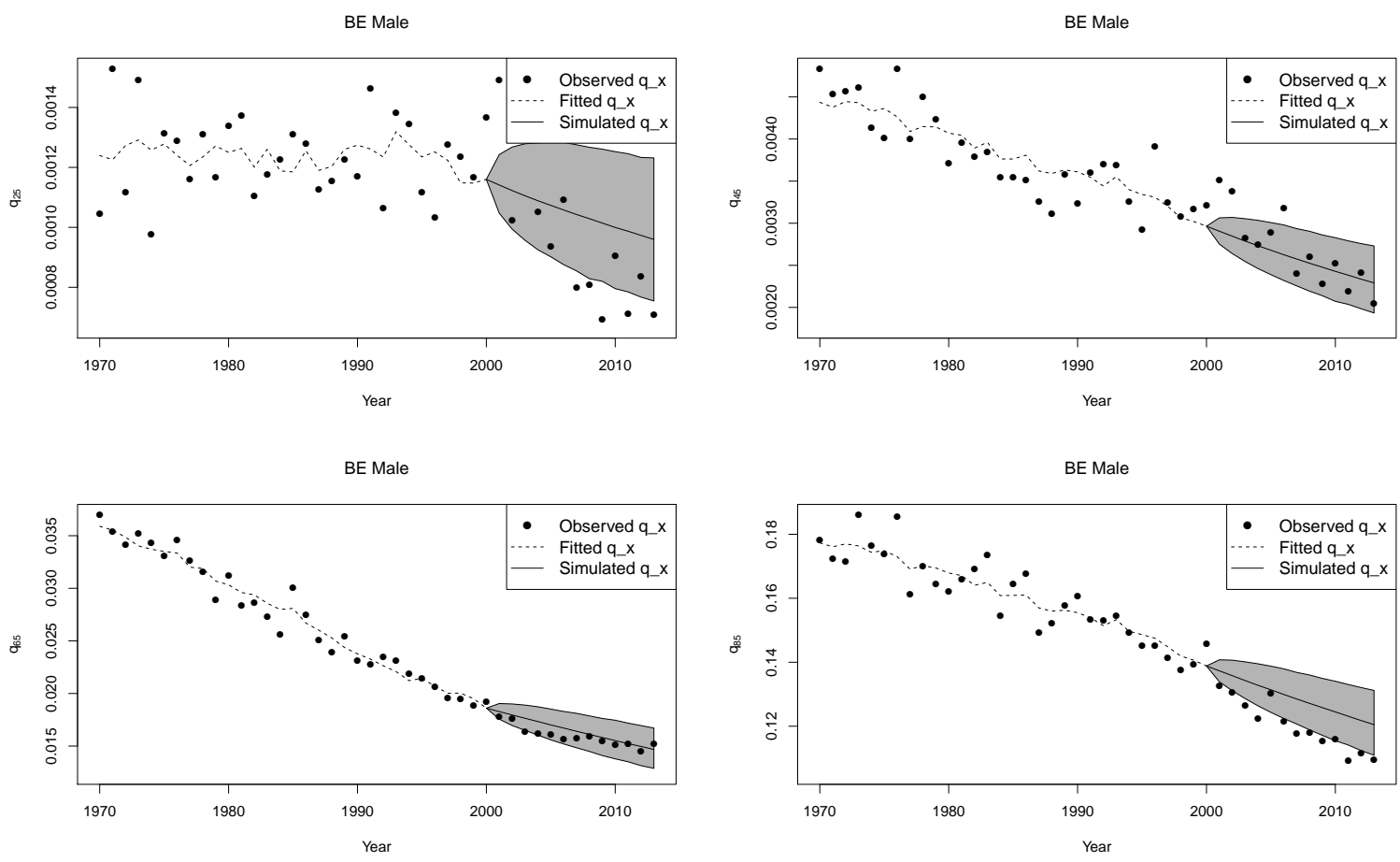

Figure 9: Back test on $q_{x, t}$, for Belgium, male data, ages 25, 45 (top row) and 65, 85 (bottom row). We calibrate on 1970-2000 data and then project 2001-2013. We plot $0.5 \%$, median and $99.5 \%$ quantile obtained from 10,000 simulations.
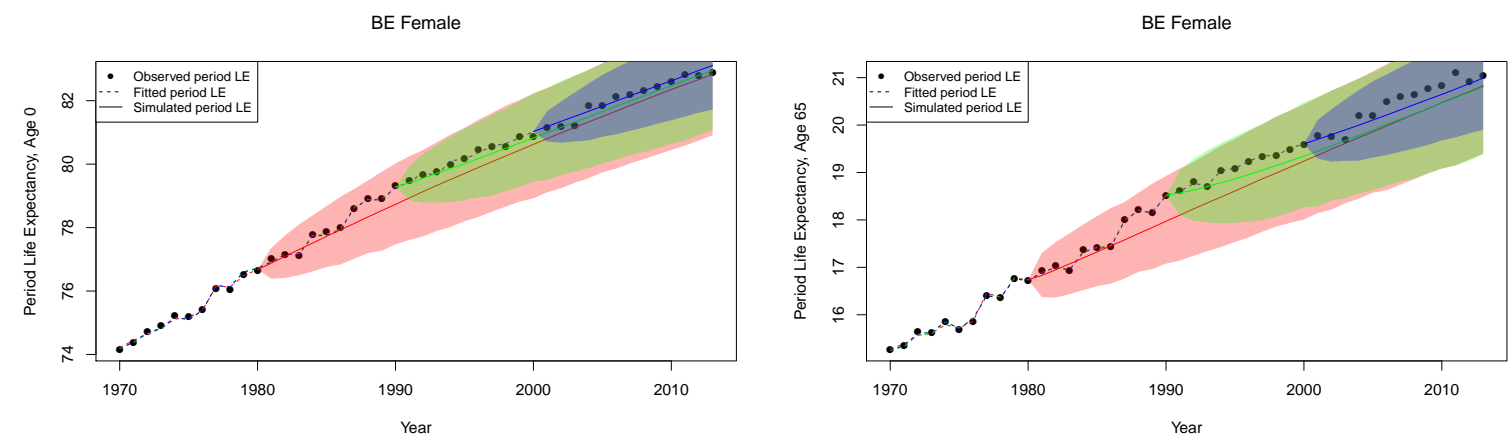

Figure 10: Back tests using calibration periods $\{1970, \ldots, 1980\}$ (red), $\{1970, \ldots, 1990\}$ (green) and $\{1970, \ldots, 2000\}$ (blue), period life expectancy for a 0 year old (left) and 65 year old (right), female data. We plot 0.5\%, median and $99.5 \%$ quantile obtained from 10,000 simulations.

Li \& Lee type and uses Belgian data, together with mortality data from a set of 13 other well selected European countries, to forecast mortality. The model follows the specifications of the 2014 projection model published by the Dutch Koninklijk Actuarieel Genootschap. Actuaries can use this model to generate scenarios for future mortality, or they can use the table of $q_{x, t}$ 's as a best estimate scenario of future mortality. We selected this model from a comparative study of a wide set of stochastic mortality models published in recent literature. The model performs well in terms of (among others) robustness, biological reasonableness, transparency, in sample 

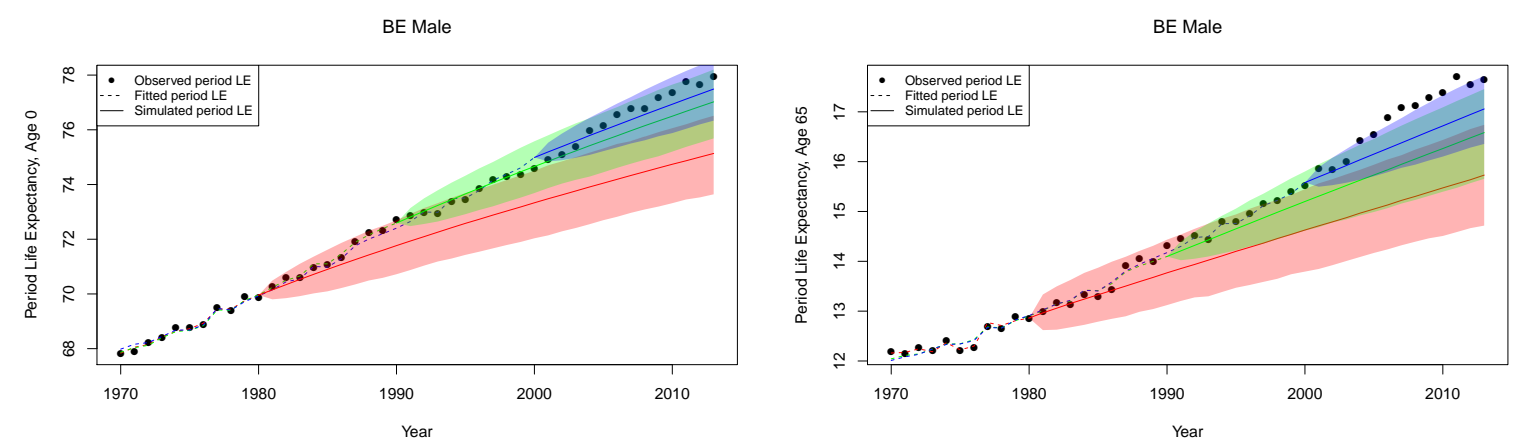

Figure 11: Back tests using calibration periods $\{1970, \ldots, 1980\}$ (red), $\{1970, \ldots, 1990\}$ (green) and $\{1970, \ldots, 2000\}$ (blue), period life expectancy for a 0 year old (left) and 65 year old (right), male data. We plot $0.5 \%$, median and $99.5 \%$ quantile obtained from 10,000 simulations.

fit and back testing.

\section{Acknowledgements}

The first author gratefully acknowledges the work of all members of the Werkgroep prognosetafels (namely: Corné van Iersel, Hok-Kwan Kan, Richard Meijer, Wilbert Ouburg, Erica Slagter, Cees Smit and prof. Anja de Waegenaere) and the Commissie sterfte onderzoek (namely: Wouter de Boer, Henk Van Broekhoven, Egbert Kromme, Tim Schulteis, prof. Michel Vellekoop, Robert de Vries, prof. Bas Werker and Marco van der Winden) of the Dutch Koninklijk Actuarieel Genootschap, see Koninklijk Actuarieel Genootschap (2014). The first author gratefully acknowledges the comments of prof. Michel Denuit and prof. Michel Vellekoop on an earlier version of this report.

\section{References}

Antonio, K. (2012). Sluiten van de periodetafel GBM/V 2005-2010. www.ag-ai.nl.

Barrieu, P., Bensusan, H., Karoui, N. E., Hillairet, C., Loisel, S., Ravanelli, C., and Salhi, Y. (2012). Understanding, modelling and managing longevity risk : key issues and main challenges. Scandinavian Actuarial Journal, 3:203-231.

Börger, M., Fleischer, D., and Kuksin, N. (2014). Modelling the mortality trend under modern solvency regimes. ASTIN Bulletin, 44(1):1-38.

Brouhns, N., Delwarde, A., and Denuit, M. (2002a). Élaboration de tables de mortalité prospectives, ou comment tarifer des rentes viagères lorsque la mortalité évolue. KVBA-ARAB Report.

Brouhns, N., Denuit, M., and Vermunt, J. (2002b). A Poisson log-bilinear regression approach to the construction of projected life tables. Insurance: Mathematics and Economics, 31:373-393.

Cairns, A., Blake, D., and Dowd, K. (2006). A two-factor model for stochastic mortality with parameter uncertainty: theory and calibration. Journal of Risk and Insurance, 73:687-718. 
Cairns, A., Blake, D., Dowd, K., Coughlan, G., Epstein, D., Ong, A., and Balevich, I. (2009). A quantitative comparison of stochastic mortality models using data from England and Wales and the United States. North American Actuarial Journal, 13(1):1-35.

Delfosse, P. and Boelen, P. (2002). Conséquences pour les rentes viagères. KVBA-ARAB Report.

Doray, L. (2008). Inference for the logistic-type models for the force of mortality. Living to 100 and beyond, SOA Monograph M-LI08-01, pages 1-18.

Federaal Planbureau (2009). Prospectieve sterftequotiënten per geslacht en uniseks. http: //www.plan.be/admin/uploaded/201002040818570.wp200918.pdf.

Federaal Planbureau (2014). Demografische vooruitzichten 2013-2060: bevolking, huishoudens en prospectieve sterftequotiënten. http://www.plan.be/admin/uploaded/ 201404150736290.FORPOP1360_10697_N.pdf.

Haberman, S. and Renshaw, A. (2011). A comparative study of parametric mortality models. Insurance: Mathematics and Economics, 48:35-55.

Jaumain, C. and Vandeschrick, C. (2012). Sterftetafel: van verstreken leeftijden naar exacte leeftijden.

Kannistö, V. (1992). Development of the oldest-old mortality, 1950-1980: evidence from 28 developed countries. Odense University Press.

Koninklijk Actuarieel Genootschap (2014). Prognosetafel AG2014. www.ag-ai.nl.

Lambrechts, K. (2001). Belgian mortality tables for the past and the next century. KVBA-ARAB Report.

Lee, R. and Carter, L. (1992). Modeling and forecasting the time series of US mortality. Journal of the American Statistical Association, 87:659-671.

Li, J. (2013). A Poisson common factor model for projecting mortality and life expectancy jointly for females and males. Population Studies: A Journal of Demography, 67(1):111-126.

Li, N. and Lee, R. (2005). Coherent mortality forecasts for a group of populations: an extension of the Lee-Carter method. Demography, 42(3):575-594.

Pitacco, E., Denuit, M., Haberman, S., and Olivieri, A. (2009). Modeling Longevity Dynamics for Pensions and Annuity Business. Oxford University Press, London.

Van Berkum, F., Antonio, K., and Vellekoop, M. (2014). The impact of multiple structural changes on mortality predictions. Scandinavian Actuarial Journal, DOI 10.1080/03461238.2014.987807. 
FACULTY OF ECONOMICS AND BUSINESS Naamsestraat 69 bus 3500

3000 LEUVEN, BELGIË

tel. + 3216326612

fax +3216326791

info@econ.kuleuven.be www.econ.kuleuven.be 